\title{
Platycodin D alleviates proliferation and extracellular matrix accumulation in TGF-betal induced pulmonary fibroblasts
}

\author{
Hou $\mathrm{HH}^{*}$, Su CC*, Hong LL, Huang HF, Tang YF \\ Pneumology Department, Nantong Hospital of TCM, Nantong, Jiangsu, China. tangyanfen2200@163.com
}

\begin{abstract}
AIM: Platycodin D (PD), an oleanane kind of triterpenoid saponin, possesses various pharmacological activities. We aimed to investigate the effects of $P D$ in pulmonary fibrosis.

METHOD: MRC-5 cells were induced by transforming growth factor-beta1 (TGF- $\beta 1$ ) to simulate the pulmonary fibrosis in vitro. Cell viability was determined using a CCK-8 kit in the absence or presence of PD. Then, the expression of proliferation-related proteins was detected using immunofluorescence assay or western blot analysis. Moreover, the levels of inflammatory factors were examined. Subsequently, the ability of cell migration was evaluated using wound healing assay. Additionally, western blot analysis was employed to determine migration- and extracellular matrix accumulation (ECM)-related proteins expression.

RESULTS: Results indicated that PD exposure significantly dose-dependently inhibited TGF- $\beta 1$ induced proliferation in MRC-5 cells. Additionally, the contents of inflammatory factors were notably inhibited with PD treatment. Furthermore, significant decrease in migration of TGF- $\beta 1$-stimulated MRC- 5 cells was observed after PD intervention. Afterwards, PD remarkably suppressed the expression of alpha smooth muscle actin ( $\alpha$-SMA), collagen I (Col I), collagen III (Col III) and E-cadherin (E-cad).

CONCLUSIONS: PD attenuated proliferation and ECM accumulation in TGF- $\beta 1$ induced lung fibroblasts, providing experimental support for the clinical application of PD in the treatment of pulmonary fibrosis (Fig. 6, Ref. 33). Text in PDF www.elis.sk

KEY WORDS: pulmonary fibrosis, platycodin D, extracellular matrix, proliferation, inflammation.
\end{abstract}

\section{Introduction}

Fibrosis is a common reaction of the body to various injuries, and the ultimate result is rapid proliferation and accumulation of connective tissue to replace normal tissue (1). Pulmonary fibrosis $(\mathrm{PF})$ is a diffuse pulmonary inflammatory disease, the pathogenesis of which mainly involves pulmonary interstitium and alveolar epithelial cells (2). It is characterized by lung fibroblast excessive proliferation, migration and activation, accumulation of a large number of extracellular matrix (ECM) and the formation of excessive collagen deposition (3). Although great progress has been observed in relation to medical therapies available for patients with $\mathrm{PF}$, there is still no effective treatment for this disease (4). Therefore, it is of great clinical and scientific significance that novel therapeutic agents associated with PF are identified.

It is generally well known that fibroblasts are key effector cells in fibrotic diseases.

Activation of fibroblasts is closely involved in the process of fibrogenesis, including that of PF (5). Upon activation, resting fi-

Pneumology Department, Nantong Hospital of TCM, Nantong, Jiangsu, China

Address for correspondence: Yanfen Tang, Nantong Hospital of TCM, 41 Jianshe Road, Nantong, Jiangsu, 226500, China.

"Contributed equally to this work broblasts acquire a myofibroblast phenotype, which is characterized by expression of contractile proteins and enhanced release of $\operatorname{ECM}(6,7)$. It is demonstrated that myofibroblasts produce profibrotic cytokines and ECM as well as accelerate lung fibrosis, remodeling and thus lung dysfunction. Transforming growth factor- $\beta 1$ (TGF- $\beta 1)$ is a multifunctional cytokine that regulates cell differentiation and growth, tissue homeostasis and inflammatory responses (8). A growing body of literature has shown that TGF- $\beta 1$ is one of the most powerful stimuli of fibroblast activation in physiologic and pathologic conditions and plays a crucial role for the persistent activation of fibroblasts in fibrotic diseases $(9,10)$. Therefore, identification of central checkpoints and mechanisms of TGF- $\beta 1$-induced fibroblast activation might offer potential therapies for fibrotic diseases.

Platycodin D (PD), an oleanane kind of triterpenoid saponin, is counted among the key bioactive constituents of the roots of Platycodon grandiflorum, which results in its extensive use for treating multiple ailments (11). To date, PD has received extensive attention due to its reported anti-inflammatory, antiviral, antifibrosis and anti-cancer roles (12-15). It has been well reported that $\mathrm{PD}$ reverses pathological myocardial hypertrophy and fibrosis in spontaneous hypertension rats (16). A study mentioned that by regulating JNK/c-JUN signal pathway, PD improves liver fibrosis and activates hepatic stellate cells (17). However, there has been no clarification made for the effects of PD on lung fibrosis. The 
145-151

present study utilized the cellular model of TGF- $\beta 1$-induced human pulmonary fibroblasts MRC-5 cells to investigate the anti-fibrotic efficacy of PD and identify the potential mechanisms.

\section{Materials and methods}

\section{Cell culture}

The human pulmonary fibroblasts MRC-5 cells were obtained from American Type Culture Collection (ATCC). Cells were cultured in RPMI-1640 with $10 \%$ fetal bovine serum (Sigma, NY) in humidified air at $37{ }^{\circ} \mathrm{C}$ in fully humidified air of $5 \% \mathrm{CO}_{2}$. MRC-5 cells were pretreated with a series of concentrations of PD $(5,10$ and $20 \mu \mathrm{M})$ for $30 \mathrm{~min}$ before stimulation with TGF- $\beta 1(5 \mathrm{ng} / \mathrm{ml})$ for $48 \mathrm{~h}$. Untreated cells were used as the control group.

\section{Cell viability assay}

Cells viability was detected using a cell counting kit-8 (CCK8 ) kit according to the manufacturer's protocol. MRC-5 cells were grown in 96-well plates when $80 \%$ confluency was reached. Cells were incubated with $10 \mu \mathrm{ccK}-8$ reagent for another $2 \mathrm{~h}$. The optical density at $450 \mathrm{~nm}$ of cells was determined using the BioTek elx800 (BioTek instruments, inc.). Experiments were performed in triplicate. perature, permeabilized with $0.1 \%$ Triton X-100 and blocked with $3 \%$ bovine serum albumin (BSA; Sigma-Aldrich; Merck KGaA) for $30 \mathrm{~min}$ at $37^{\circ} \mathrm{C}$. Following probed with primary antibodies recognizing PCNA at $4{ }^{\circ} \mathrm{C}$ overnight, cells were incubated with the secondary antibody (Beyotime Institute of Biotechnology) for $2 \mathrm{~h}$ at room temperature. Nuclei were stained with DAPI (Roche Diagnostics) in the dark at room temperature for $5 \mathrm{~min}$, and images were taken under an Olympus microscope (magnification, x200; Tokyo, Japan).

\section{Western blot analysis}

Protein samples were extracted from MRC-5 cells for western blot analysis. The protein concentration of each extract in the supernatant was determined using a BCA Protein Assay Kit (Beyotime Institute of Biotechnology, Shanghai, China). Fifty microgram of the total protein was exposed to $10 \%$ sodium dodecyl sulfate-polyacrylamide gel electrophoresis (SDS-PAGE) and subsequently transferred onto PVDF membranes. The membranes were sealed with $5 \%$ non-fat milk for $1 \mathrm{~h}$ at room temperature, then incubated with probed with specific primary antibodies (Cell Signaling Technology; Boston, MA, USA) overnight at $4{ }^{\circ} \mathrm{C}$. Following washing with TBST for three times, the membranes were incubated with secondary antibody (Cell Signaling Technology,

\section{Scratch wound healing assay}

For the wound healing assay, cells were seeded in a six-well plate and incubated at $37{ }^{\circ} \mathrm{C}$ until $80 \%$ confluence. A straight scratch on cell monolayers was gently created by a sterile $200-\mu 1$ pipette tip. Cells were washed twice with PBS to remove cell debris. Then, media was replaced with serum-free medium. The average distance of cells migrating into wound surface was tested after $24 \mathrm{~h}$ of wounding under an inverted microscope (Olympus, Japan).

Detection of inflammatory cytokines levels

The concentrations of inflammatory factors including tumor necrosis factor (TNF)- $\alpha$, interleukin (IL)- 6 and IL- $1 \beta$ were examined using enzyme linked immunosorbent assay kits on the basis of the manufacturer's protocols. Above-mentioned kits were the products of Shanghai Xitang Biotechnology Co., Ltd. (Shanghai, China).

\section{Immunofluorescence assay}

MRC-5 cells were grown on sterile glass slides for $24 \mathrm{~h}$ in 6-well plates to reach 80-90\% confluency. Subsequently, cells were serum starved for $12 \mathrm{~h}$. After treatment with PD and TGF- $\beta 1$, MRC-5 cells were washed with PBS, immobilized with $4 \%$ paraformaldehyde for $15 \mathrm{~min}$ at room tem-
A

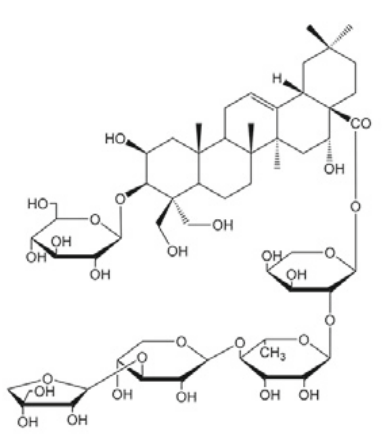

(B)

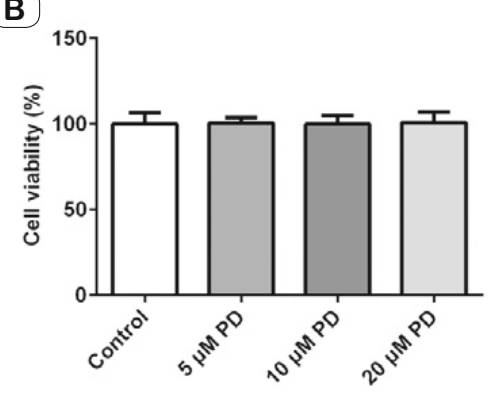

(C)

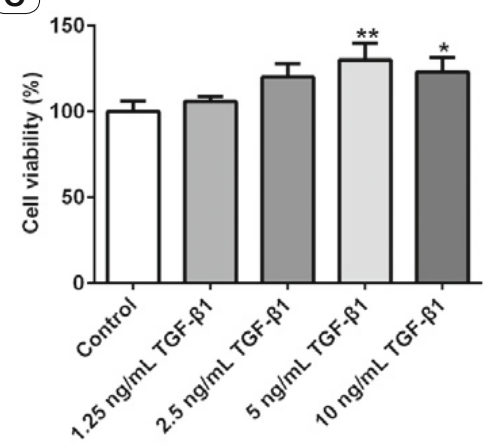

(D)

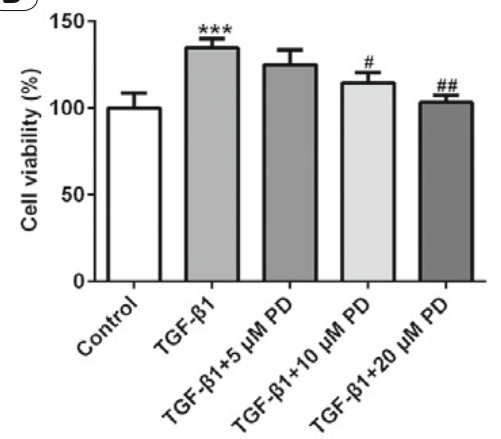

Fig. 1. PD alleviated the increase of cell viability induced by TGF- $\beta 1$. (A) The chemical structural formula of PD. Cell viability was detected using a CCK-8 kit after treatment with (B) a series of concentrations of PD or (C) TGF- $\beta 1 .{ }^{*} p<0.05$ and ${ }^{* *} p<0.01$ vs control. (D) PD alleviates the increase of MRC-5 cell viability induced by TGF- $\beta 1$. ${ }^{* * *} p<0.001$ vs control; ${ }^{\#} p$ $<0.05,{ }^{\#} p<0.01$ vs TGF- $\beta 1$. PD - Platycodin D; TGF- $\beta 1$ - transforming growth factor- $\beta 1$; CCK-8 - cell counting kit-8. 


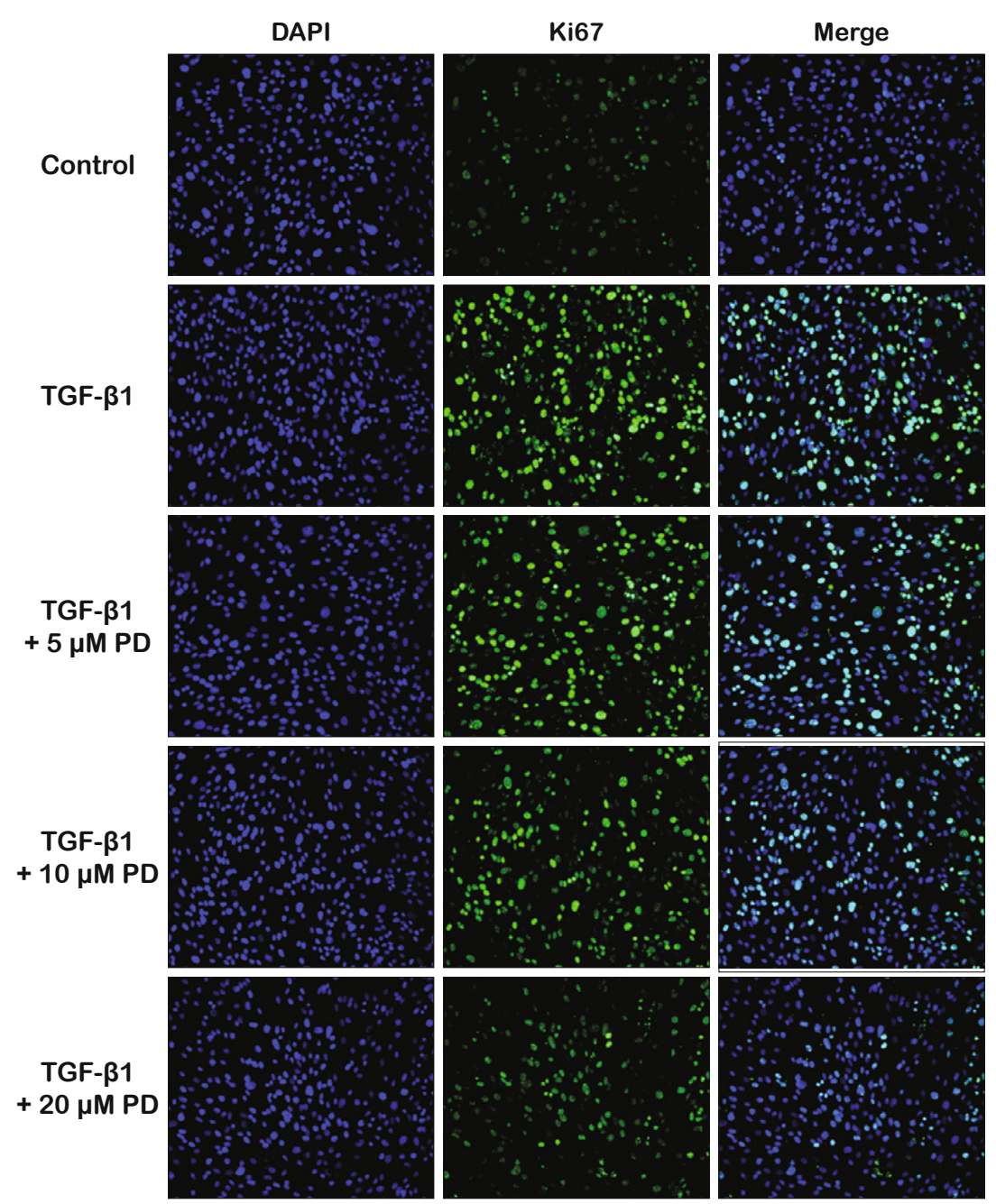

Fig. 2. PD downregulated the level of Ki67 in TGF- $\beta 1$-induced MRC-5 cells. The expression of Ki67 was detected using immunofluorescence assay. PD - Platycodin D; TGF- $\beta 1$ - transforming growth factor- $\beta 1$.

Boston, MA, USA). The immunoreactive protein bands on the membrane were visualized using the Odyssey Infrared Imaging System (LI-COR Biosciences). GAPDH was applied to detect the relative protein expression.

\section{Statistical analysis}

Data are expressed as mean \pm standard deviation and were analyzed using GraphPad Prism version 6.0 (GraphPad Software, Inc.). T-test was utilized to pairwise comparison and the comparisons among multiple groups were conducted using analysis of variance (ANOVA) followed by Turkey's post hoc test. The difference of experimental data was statistically significant when $\mathrm{p}<0.05$.

\section{Results}

PD alleviates proliferation of MRC-5 cells stimulated by TGF- $\beta 1$

The chemical structural formula of PD was displayed in Figure 1A. Cell viability was detected using a CCK-8 kit after treat- ment with a series of different concentrations of PD. As observable in Figure 1B, there were no significant effects of cell viability in response to $\mathrm{PD}$ challenge relative to the control group. However, significantly elevated viability of MRC-5 cells was found in the TGF- $\beta 1$-treated groups, especially in the $5 \mathrm{ng} / \mathrm{mL}$ TGF- $\beta 1$ group, which was selected for the following experiments (Fig. 1C). Afterwards, PD markedly reduced the increase of cell viability induced by TGF- $\beta 1$ in a dose-dependent manner (Fig. 1D). Then, the level of Ki67 was determined using immunofluorescence assay. As presented in Figure 2, Ki67 expression was remarkably dose-dependently downregulated in TGF- $\beta 1$-stimulated MRC-5 cells after PD exposure. Consistently, Ki67 and proliferating cell nuclear antigen (PCNA) expression levels evaluated by western blot analysis shown the same results with the immunofluorescence assay (Fig. 3). These findings provided a clue that PD attenuates proliferation of MRC- 5 cells induced by TGF- $\beta 1$.

\section{$P D$ attenuates inflammation in TGF- $\beta 1$ - induced MRC-5 cells}

Subsequently, the effects of PD on inflammatory response in MRC-5 cells stimulated by TGF- $\beta 1$ was examined using the ELISA kits, respectively. As exhibited in Figures $4 \mathrm{~A}-\mathrm{C}$, TGF- $\beta 1$ induction significantly enhanced the contents of TNF- $\alpha$, IL$1 \beta$ and IL- 6 relative to the control group. As comparison to the TGF- $\beta 1$-stimulated group, $\mathrm{PD}$ intervention dose-dependently decreased the levels of above-mentioned inflammatory factors. These observations reveal that PD suppresses inflammation in TGF- $\beta 1$-induced MRC-5 cells.

\section{$P D$ inhibits TGF- $\beta 1$-induced migration of MRC-5 cells}

To investigate the function of PD on migration of MRC-5 cells, scratch wound healing assay was employed to perform the experiment. As shown in Figures $5 \mathrm{~A}$ and B, TGF- $\beta 1$ stimulation notably promoted migration of MRC- 5 cells compared with the control. However, remarkably decreased cell migration ability was noticed when PD exposure. Simultaneously, the expression of migration-related proteins including matrix metalloproteinase-2 (MMP2) and MMP9 was determined using western blot analysis. Results shown in Figure 5C revealed that PD treatment dramatically downregulated the expression of both MMP2 and MMP9 in a dose-dependent manner relative to the TGF- $\beta 1$ stimulation alone. Overall, these data suggest that PD suppresses migration in TGF- $\beta 1$-induced MRC- 5 cells. 
145-151
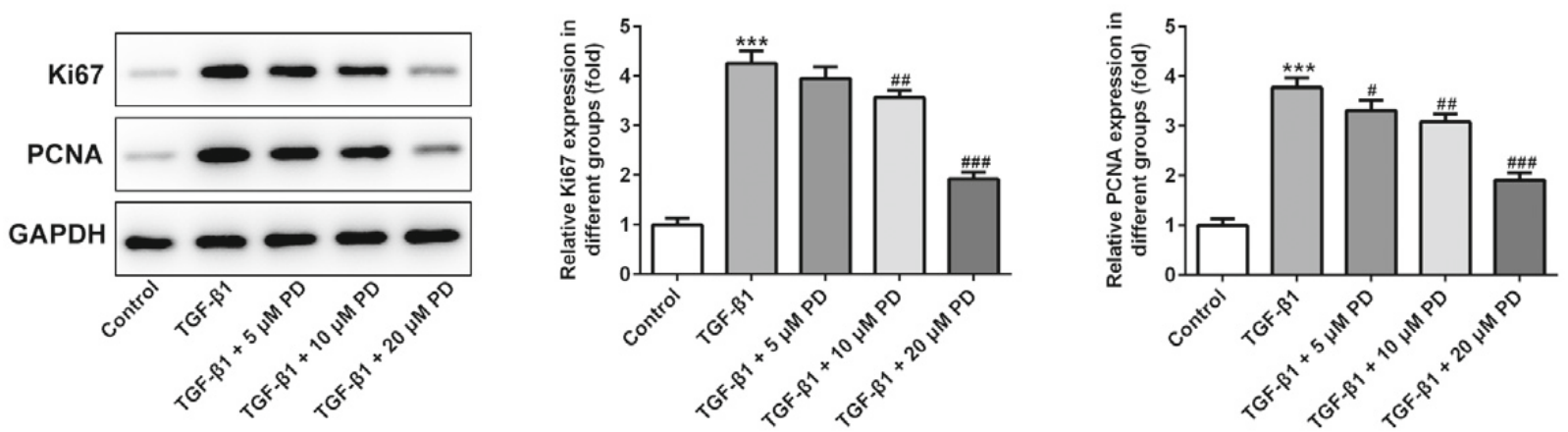

Fig. 3. PD decreased the expression of Ki67 and PCNA in TGF- 11 -induced MRC-5 cells. Both Ki67 and PCNA expression were determined using western blot analysis. ${ }^{* * * *} p<0.001$ vs control; ${ }^{\#} p<0.05,{ }^{\#} p<0.01,{ }^{\# \#} p<0.001$ vs TGF- $\beta 1$. PD - Platycodin D; TGF- $\beta 1$ - transforming growth factor- $\beta 1$; PCNA - proliferating cell nuclear antigen.

(A)

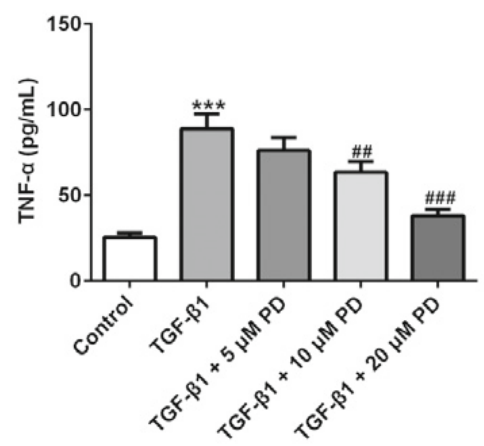

(B)

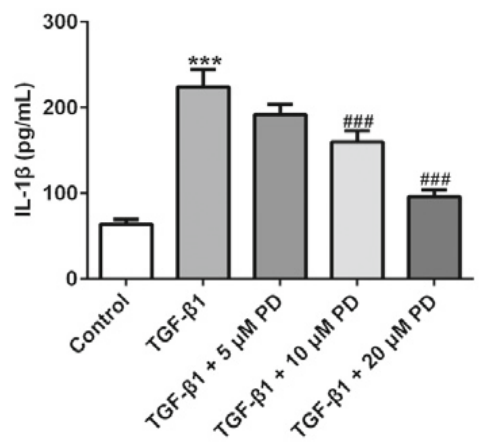

(C)

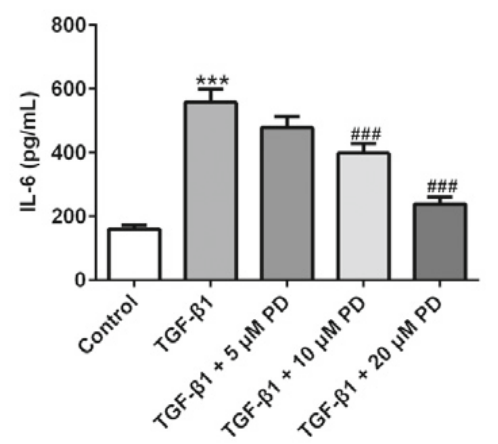

Fig. 4. PD inhibited inflammation in TGF- $\beta 1$-stimulated MRC-5 cells. The contents of inflammatory factors including (A) TNF- $\alpha$, (B) IL-1 $\beta$ and (C) IL-6 were examined using ELISA kits, respectively. ${ }^{* * *} p<0.001$ vs control; ${ }^{\#} p<0.01$, ${ }^{\# \#} p<0.001$ vs TGF- $\beta 1$. PD - Platycodin D; TGF- $\beta 1$

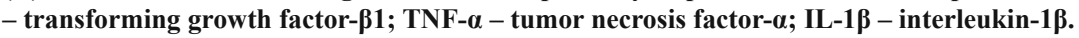

PD blocked the expression of ECM-related proteins in TGF- $\beta 1$ induced MRC-5 cells

To explore the effects of PD on ECM deposition, the expression of ECM-associated key proteins was determined using western blot analysis. As it is observable from Figure 6, TGF- $\beta 1$ induction led to significantly elevated expression of alpha smooth muscle actin $(\alpha$-SMA), collagen I (Col I), collagen III (Col III) and E-cadherin (E-cad) compared with the control group. By contrast, PD exposure markedly reversed the influence of TGF- $\beta 1$ on expression of fore-mentioned proteins. Through the above findings we proved that PD inhibits ECM deposition in TGF- $\beta 1$-induced MRC- 5 cells.

\section{Discussion}

$\mathrm{PF}$ is a progressive and fatal lung disease in which median survival time following diagnosis is $2-5$ years (18). It has been well reported that the incidence of $\mathrm{PF}$ is increasing in recent years (19). Therefore, PF has been a serious threat to human health. The present study used MRC- 5 cells stimulated by TGF- $\beta 1$ to simulate the PF model in vitro. Our findings demonstrated that PD effec- tively attenuates the excessive proliferation, migration, collagen deposition and ECM expression in TGF- $\beta 1$ induced MRC- 5 cells.

Accumulating evidence shows that the abnormal behavior of alveolar epithelial cells provokes fibroblast proliferation, migration and differentiation (10). TGF- $\beta 1$ is considered to be one of the most crucial factors responsible for $\mathrm{PF}$, with the abilities to promote fibroblast proliferation, migration and the synthesis of connective tissue components (20). It's widely accepted that widely distributed fibroblasts in the structures of the lungs play a key role in the process of fibrosis (21). Existing study has shown that fibroblasts isolated from lung tissues of patients with PF exhibited marked variability in proliferative property under some stimulation (22). Inhibition of fibroblast proliferation protects against bleomycininduced pulmonary fibrosis (23). Current evidence suggests that chronic inflammation and wound healing are the dominant cause of PF accompanied by fibroblasts activity (24). Compelling evidence indicates that PF occurs as the result of aberrant wound healing responses, and fibroblasts are key players during the pathogenesis (25). And TGF- $\beta 1$ stimulation induced lung fibroblasts to secrete a large amount of inflammatory cytokines (26). A growing body 


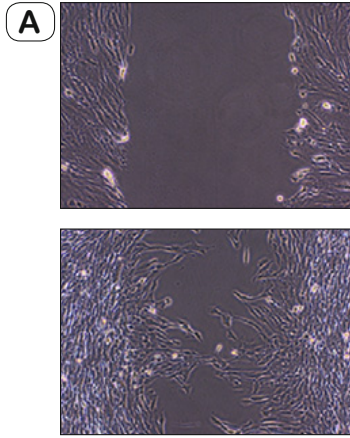

Control
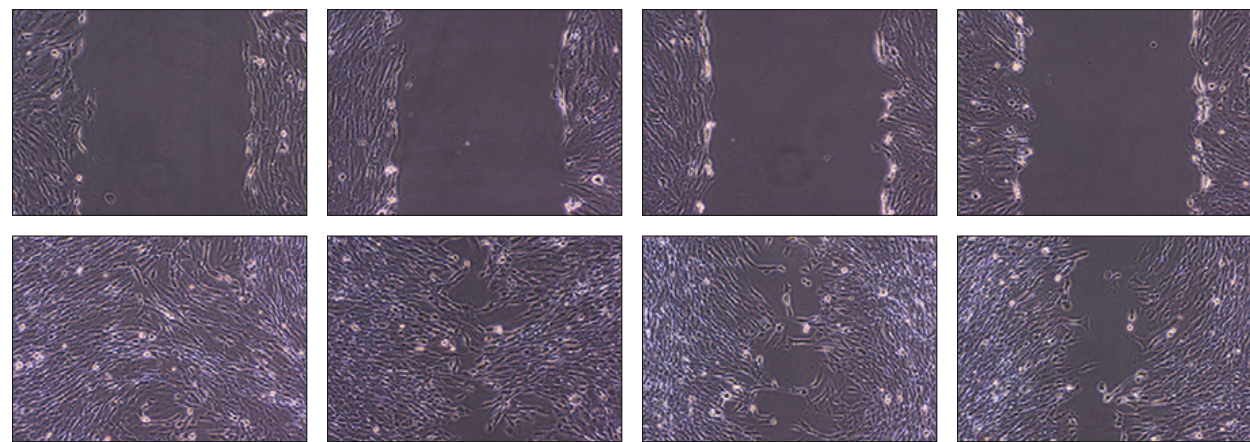

TGF- $\beta 1$

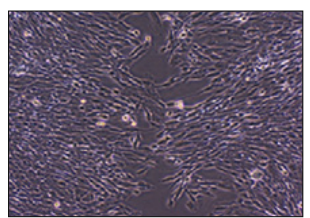

TGF- $\beta 1$

$+5 \mu \mathrm{M}$ PD

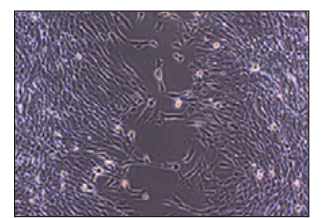

TGF- $\beta 1$

$+10 \mu \mathrm{M}$ PD

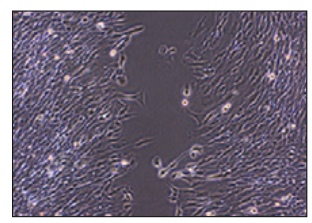

TGF- $\beta 1$

$+20 \mu \mathrm{M}$ PD

(B)

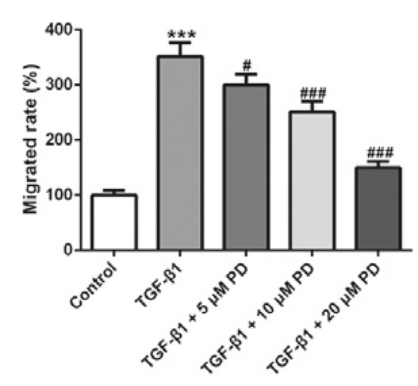

(C)

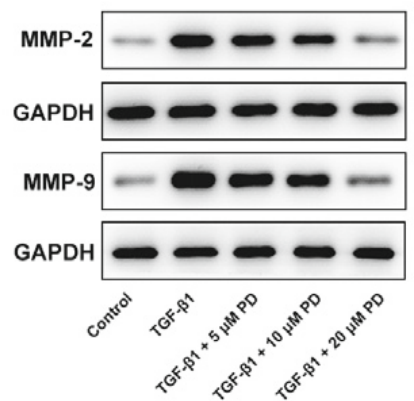

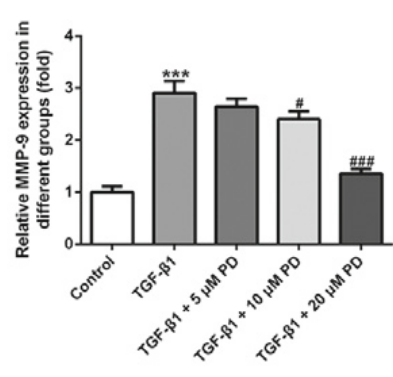

Fig. 5. PD suppressed migration in TGF- $\beta 1$-induced MRC-5 cells. (A and B) scratch wound healing assay was employed to measure the migration of MRC-5 cells. (C) The expression of MMP2 and MMP9 was detected using western blot analysis. ${ }^{* * * *} p<0.001$ vs control; ${ }^{\#} p<0.05$, ${ }^{\# \#} p$

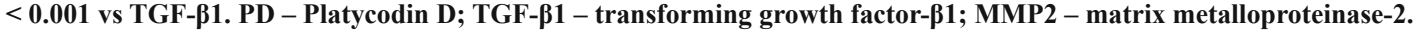
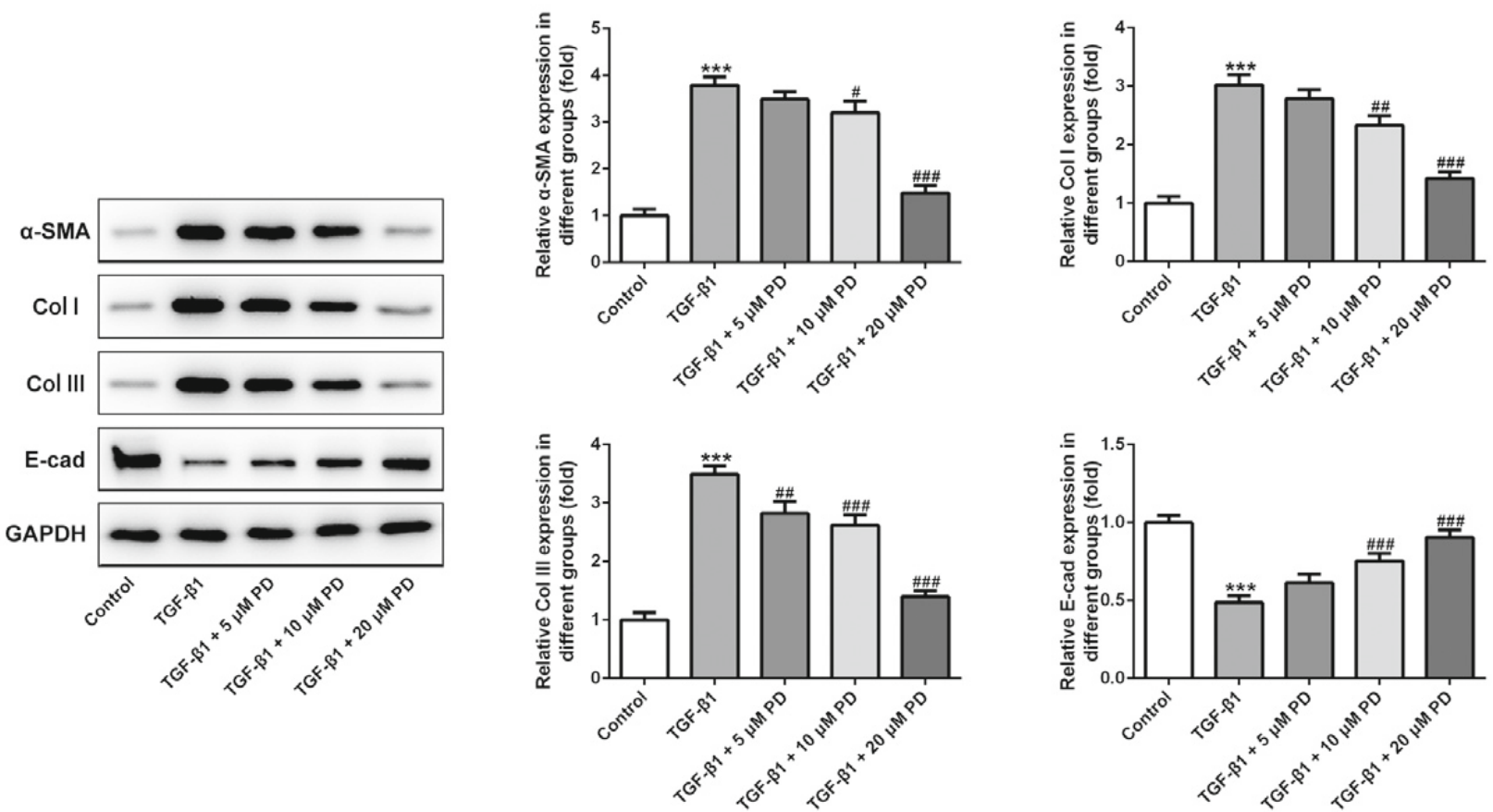

Fig. 6. PD blocked the expression of ECM-related proteins in TGF- $\beta 1$-induced MRC-5 cells. Western blot analysis was applied for evaluating the expression of $\alpha$-SMA, Col I, Col III and E-cad. ${ }^{\text {**t* }}$ p $<0.001$ vs control; ${ }^{\#} p<0.05,{ }^{\#} p<0.01,{ }^{\# \#} p<0.001$ vs TGF- $p 1$. PD - Platycodin D; TGF- $\beta 1$ - transforming growth factor- $\beta 1 ; \alpha-S M A$ - alpha smooth muscle actin; Col I - collagen I; Col III - collagen III; E-cad - E-cadherin. 


\section{5-151}

of evidence suggests that PD can suppress proliferation and migration in a variety of human cancers, such as myeloma, breast cancer and non-small cell lung cancer $(13,27,28)$. Moreover, PD alleviates acute lung injury through inhibition of inflammation $(29,30)$. This study indicated that PD treatment significantly suppressed proliferation, inflammation and migration of TGF- $\beta 1$ induced MRC-5 cells, suggesting a potential therapeutic effects of PD on pulmonary fibroblasts.

Pulmonary fibrosis is characterized by progressive ECM deposition, destruction of the lung tissue structure and ultimate loss of lung function. Mounting evidence supports the notion that the high levels of collagen and $\alpha$-SMA in lung fibrotic lesions generated by activated fibroblasts are closely related to fibrotic lung disease (31). These activated fibroblasts have the characteristics of mobility and contraction as well as synthesize and secrete a plenty of ECM, resulting in the formation of collagenfiber networks, which together affect respiratory mechanics (32). MRC-5 cells, which retain the features of activated lung fibroblasts including $\alpha$-SMA expression and the excessive secretion of ECM, are therefore an attractive cell line for use in pulmonary fibrosis research (33). Investigation of methods of interfering with fibroblast activation may thus provide novel therapeutic strategies for the treatment of pulmonary fibrosis. Reports have demonstrated previously that PD reverses pathological myocardial hypertrophy and fibrosis in spontaneous hypertension rats (16). Additionally, PD improves liver fibrosis and activates hepatic stellate cells by regulating JNK/c-JUN signal pathway (17). In the present study, the expression of $\alpha$-SMA, Col I, Col III and E-cad was markedly downregulated after PD exposure, suggesting that PD treatment suppresses ECM accumulation in TGF- $\beta 1$-induced MCR-5 cells.

\section{Conclusion}

Taken together, on the basis of the intrinsic regulator effects of $\mathrm{PD}$ on the proliferation, migration, inflammation and ECM accumulation in TGF- $\beta 1$-induced MRC- 5 cells, PD is a qualified candidate as a therapeutic agent for the treatment of pulmonary fibrosis. Our study provides experimental supports for the clinical application of PD in the treatment of this disease. However, the lack of animal experiments is a limitation of the present study, which will be investigated in future research.

\section{References}

1. Wynn TA. Cellular and molecular mechanisms of fibrosis. J Pathol 2008; 214: 199-210.

2. Meyer KC. Pulmonary fibrosis, part I: epidemiology, pathogenesis, and diagnosis. Exp Rev Respir Med 2017; 11: 343-359.

3. Hu X, Xu Q, Wan H et al. PI3K-Akt-mTOR/PFKFB3 pathway mediated lung fibroblast aerobic glycolysis and collagen synthesis in lipopolysaccharide-induced pulmonary fibrosis. Lab Invest 2020; 100: 801-811.

4. Kyung SY, Kim DY, Yoon JY et al. Sulforaphane attenuates pulmonary fibrosis by inhibiting the epithelial-mesenchymal transition. BMC Pharmacol Toxicol 2018; 19: 13.
5. Wynn TA, Ramalingam TR. Mechanisms of fibrosis: therapeutic translation for fibrotic disease. Nat Med 2012; 18: 1028-1040.

6. Hoyles RK, Derrett-Smith EC, Khan K et al. An essential role for resident fibroblasts in experimental lung fibrosis is defined by lineagespecific deletion of high-affinity type II transforming growth factor beta receptor. Am J Respir Crit Care Med 2011; 183: 249-26.

7. Hinz B, Phan SH, Thannickal VJ, Galli A, Bochaton-Piallat ML, Gabbiani G. The myofibroblast: one function, multiple origins. Am J Pathol 2007; 170: 1807-1816.

8. Biernacka A, Dobaczewski M, Frangogiannis NG. TGF-beta signaling in fibrosis. Growth Factors 2011; 29: 196-202.

9. Fix C, Carver-Molina A, Chakrabarti M, Azhar M, Carver W. Effects of the isothiocyanate sulforaphane on TGF-beta1-induced rat cardiac fibroblast activation and extracellular matrix interactions. J Cell Physiol 2019; 34: 13931-13941.

10. Pan R, Zhang Y, Zheng M, Zang B, Jin M. Hydroxysafflor Yellow A Suppresses MRC-5 Cell Activation Induced by TGF-beta1 by Blocking TGF-beta1 Binding to TbetaRII. Front Pharmacol 2017; 8: 264.

11. Fu Y, Xin Z, Liu B et al. Platycodin D Inhibits Inflammatory Response in LPS-Stimulated Primary Rat Microglia Cells through Activating LXRalpha-ABCA1 Signaling Pathway. Front Immunol 2017; 8: 1929.

12. Sun F, Liu FG. Platycodin D inhibits MPP+-induced inflammatory response in BV-2 cells through the TLR4/MyD88/NF-kappa B signaling pathway. J Recept Signal Transduct: 7.

13. Wu D, Zhang W, Chen Y, Ma H, Wang MC. Platycodin D inhibits proliferation, migration and induces chemosensitization through inactivation of the NF-kappa B and JAK2/STAT3 pathways in multiple myeloma cells. Clin Exp Pharmacol Physiol 2019; 46: 1194-1200.

14. Kim JW, Park SJ, Lim JH et al. Triterpenoid Saponins Isolated from Platycodon grandiflorum Inhibit Hepatitis C Virus Replication. Evid Based Complement Alternat Med 2013; 560417.

15. Shi C, Li Q, Zhang X. Platycodin D Protects Human Fibroblast Cells from Premature Senescence Induced by $\mathrm{H} 2 \mathrm{O} 2$ through Improving Mitochondrial Biogenesis. Pharmacology 2020; 1-11.

16. Lin YC, Lin YC, Kuo WW et al. Platycodin D Reverses Pathological Cardiac Hypertrophy and Fibrosis in Spontaneously Hypertensive Rats. Am J Chin Med 2018; 46: 537-549.

17. Liu YM, Cong S, Cheng $Z$ et al. Platycodin D alleviates liver fibrosis and activation of hepatic stellate cells by regulating JNK/c-JUN signal pathway. Eur J Pharmacol 2020; 876: 172946.

18. O'Dwyer DN, Ashley SL, Moore BB. Influences of innate immunity, autophagy, and fibroblast activation in the pathogenesis of lung fibrosis. Am J Physiol Lung Cell Mol Physiol 2016; 311: L590-601.

19. Raghu G. Idiopathic pulmonary fibrosis: guidelines for diagnosis and clinical management have advanced from consensus-based in 2000 to evidence-based in 2011. Eur Respir J 2011; 37: 743-746.

20. Fernandez IE, Eickelberg $\boldsymbol{O}$. The impact of TGF-beta on lung fibrosis: from targeting to biomarkers. Proc Am Thorac Soc 2012; 9: 111-116.

21. Penke LR, Speth JM, Dommeti VL, White ES, Bergin IL, PetersGolden M. FOXM1 is a critical driver of lung fibroblast activation and fibrogenesis. J Clin Invest 2018; 128: 2389-2405.

22. Yanai $\mathbf{H}$, Shteinberg A, Porat $\mathbf{Z}$ et al. Cellular senescence-like features of lung fibroblasts derived from idiopathic pulmonary fibrosis patients. Aging (Albany NY) 2015; 7: 664-672. 
23. Yin WL, Han J, Zhang ZJ, Han ZM, Wang SY. Aloperine Protects Mice against Bleomycin-induced Pulmonary Fibrosis by Attenuating Fibroblast Proliferation and Differentiation. Scientific Reports 2018; 8: 10.

24. Nie Y, Yang Y, Zhang J et al. Shikonin suppresses pulmonary fibroblasts proliferation and activation by regulating Akt and p38 MAPK signaling pathways. Biomed Pharmacother 2017; 95: 1119-1128.

25. Aggarwal S, Mannam P, Zhang J. Differential regulation of autophagy and mitophagy in pulmonary diseases. Am J Physiol Lung Cell Mol Physiol 2016; 311: L433-452.

26. Zhang P, Liu J, Zong R. Triptolide protects against TGF-beta1-induced pulmonary fibrosis by regulating FAK/calpain signaling. Exp Ther Med 2019; 18: 4781-4789.

27. Chun J, Kim YS. Platycodin D inhibits migration, invasion, and growth of MDA-MB-231 human breast cancer cells via suppression of EGFR-mediated Ala and MAPK pathways. Chem Biol Interact 2013; 205 : 212-221.

28. Seo YS, Kang OH, Kong R et al. Polygalacin D induces apoptosis and cell cycle arrest via the PI3K/Akt pathway in non-small cell lung cancer. Oncol Rep 2018; 39: 1702-1710.
29. Tao WW, Su Q, Wang HQ et al. Platycodin D attenuates acute lung injury by suppressing apoptosis and inflammation in vivo and in vitro. Int Immunopharmacol 2015; 27: 138-147.

30. Gao W, Guo Y, Yang HX. Platycodin D protects against cigarette smoke-induced lung inflammation in mice. Int Immunopharmacol 2017; 47: 53-58.

31. Bhogal RK, Stoica CM, McGaha TL, Bona CA. Molecular aspects of regulation of collagen gene expression in fibrosis. J Clin Immunol 2005; 25: 592-603.

32. Hinz B. The myofibroblast: paradigm for a mechanically active cell. J Biomech 2010; 43: 146-155.

33. Ding $\mathbf{S}$, Chen $\mathbf{G}$, Zhang $\mathbf{W}$ et al. MRC-5 fibroblast-conditioned medium influences multiple pathways regulating invasion, migration, proliferation, and apoptosis in hepatocellular carcinoma. J Transl Med 2015; 13: 237 .

Received June 23, 2020. Accepted July 14, 2020. 